$\Rightarrow$ ULTRAFAST SPECTROSCOPY

\title{
Real-time bond formation
}

Nuclear rearrangements during chemical reactions occur on the femtosecond-picosecond timescale. Ultrafast spectroscopy techniques have been used to observe photo-induced bond dissociations and isomerizations in real time for simple unimolecular reactions. However, these approaches become far more challenging to be applied for the study of intermolecular reactions. Writing in the Journal of the American Chemical Society, Tahei Tahara and colleagues use time-resolved impulsive stimulated Raman spectroscopy (TR-ISRS) to investigate the structural changes occurring during formation of a covalent bond in the dicyanoaurate anion trimer. Studying the molecular vibrations in the terahertz region enabled Tahara and co-workers to propose a new reaction mechanism.

Photo-induced nuclear and electronic dynamics in single molecules can be investigated by using precise pulses that target these specific dynamics with femtosecond accuracy. However, in the case of photo-induced bond formations, these dynamics occur over random timescales, complicating their

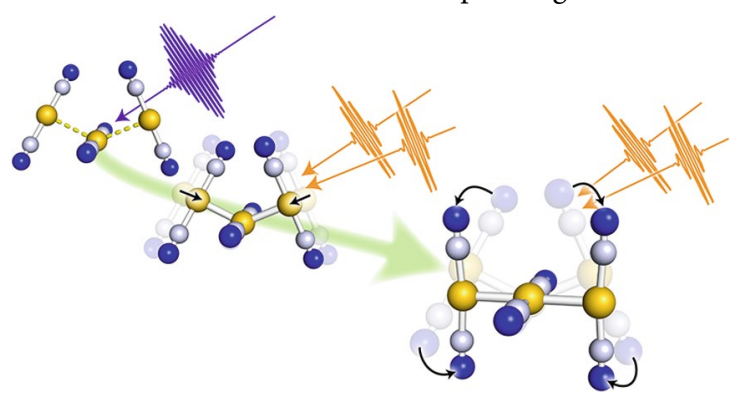

Credit: Adapted with permission from Kuramochi et al. (2019), ACS real-time observation. Oligomers of dicyanoaurate anion represent an exception because they respond instantaneously to the pump pulse. In the ground state, $\left[\mathrm{Au}(\mathrm{CN})_{2}^{-}\right]$are loosely bound in solution through $\mathrm{Au}-\mathrm{Au}$ interactions, which are of similar strength to hydrogen bonds in water. Electronic excitations induce structural changes in these ions - for example, the initial bent loose geometry of the trimer changes into a linear staggered one - with the weak aurophilic interactions becoming stronger covalent bonds. The dynamics of this reaction have previously been addressed using ultrafast optical absorption and $\mathrm{X}$-ray solution scattering. However, these experiments led to different conclusions regarding the reaction mechanism.

TR-ISRS targets specific vibrational modes of the reacting system, providing potentially more selective information than X-ray scattering in a complex solution. Compared with most ultrafast optical pump-probe techniques, TR-ISRS can access lower frequency modes that directly characterize the reaction coordinate. "We initiated the photoreaction of the sample with a femtosecond UV pump pulse that prepares a triplet excited state of the trimer. Subsequently, we induced coherent vibrations in the excited trimer using an ultrashort, visible 10 -fs pulse and recorded them using a third, visible 10 -fs probe pulse in the time domain. Fourier transform of the recorded time-domain vibrational signal provided the Raman spectrum as a function of time," explains Hikaru Kuramochi, co-author of the work. TR-ISRS can track molecular vibrations and lowfrequency collective modes down to the terahertz region. Tahara and co-workers followed the $\mathrm{Au}-\mathrm{Au}$ breathing mode of $\left[\mathrm{Au}(\mathrm{CN})_{2}^{-}\right]_{3}$ that evolves according to structural changes of the Au backbone during the bond formation. The frequency of this breathing mode upshifts and its intensity increases with a 3-ps time constant, indicating that the structural changes occur when the system is in the triplet $\mathrm{T}_{1}$ state. These results support the proposals based on transient absorption experiments but contradict those from X-ray scattering studies. The discrepancy in the latter is attributed to oligomers other than the trimer. "Our data provide a unified picture of how nuclear coordinates rearrange upon the bond formation in dicyanoaurate anion trimer: the bond contraction occurs first and this is followed by the change from a bent to linear structure," says Kuramochi.

The additional mechanistic understanding was provided by probing vibrational modes in the terahertz region that respond to the structural changes during the chemical reaction. More generally, this study shows how a range of stateof-the-art ultrafast techniques, which are increasingly accessible, need to be combined to fully understand even relatively simple chemical processes.

Margherita Citroni, Associate Editor, Nature Communications

ORIGINAL ARTICLE Kuramochi, H. et al. Tracking photoinduced $\mathrm{Au}$-Au bond formation through transient terahertz vibrations observed by femtosecond time-domain raman spectroscopy. J. Am. Chem. Soc. 141, 19296-19303 (2019) 